\title{
Corrigendum to "Nutrient supply in the Southern East China Sea after Typhoon Morakot"
}

\author{
by Chin-Chang Hung ${ }^{1,2,3}$, Chih-Ching Chung ${ }^{3}$, Gwo-Ching Gong,4, ${ }^{3,4}$, Sen Jan ${ }^{5}$, \\ Yaling Tsai $^{5}$, Kuo-Shu Chen ${ }^{1}$, Wen Chen Chou ${ }^{3}$, Ming-An Lee ${ }^{6}$, Yi Chang ${ }^{7}$, \\ Meng-Hsien Chen ${ }^{8}$, Wen-Rong Yang ${ }^{9}$, Chiung-Jung Tseng' ${ }^{9}$ and \\ Glen Gawarkiewicz ${ }^{10}$
}

The authors regret that some errors occurred in the Figure 6 published with incorrect data in our recent article (Hung et al. 2013: 145). The corrected data are presented in the revised Figure 6 below. The conclusions drawn in the original article are still supported by the revised results, though some estimates have been corrected.

Continued on the next page

1. Institute of Marine Geology and Chemistry, and Asia-Pacific Ocean Research Center, National Sun Yat-sen University, Kaohsiung, 80424, Taiwan

2. Corresponding author email: cchung@mail.nsysu.edu.tw

3. Institute of Marine Environmental Chemistry and Ecology, National Taiwan Ocean University, Keelung, 20224, Taiwan

4. Center of Excellence for Marine Bioenvironment and Biotechnology, National Taiwan Ocean University

5. Institute of Oceanography, National Taiwan University, Taipei, Taiwan

6. Department of Environmental Biology and Fisheries Science, National Taiwan Ocean University

7. Institute of Ocean Technology and Marine Affairs, National Cheng Kung University, Tainan, Taiwan

8. Department of Marine Biotechnology and Resources, National Sun Yat-sen University, Kaohsiung, Taiwan

9. Taiwan Ocean Research Institute, National Applied Research Laboratories, Kaohsiung, Taiwan

10. Woods Hole Oceanographic Institution, Woods Hole, MA, USA

(C) 2013 Chin-Chang Hung, Chih-Ching Chung, Gwo-Ching Gong, Sen Jan, Yaling Tsai, Kuo-Shu Chen, Wen Chen Chou, Ming-An Lee, Yi Chang, Meng-Hsien Chen, Wen-Rong Yang, Chiung-Jung Tseng and Glen Gawarkiewicz. 


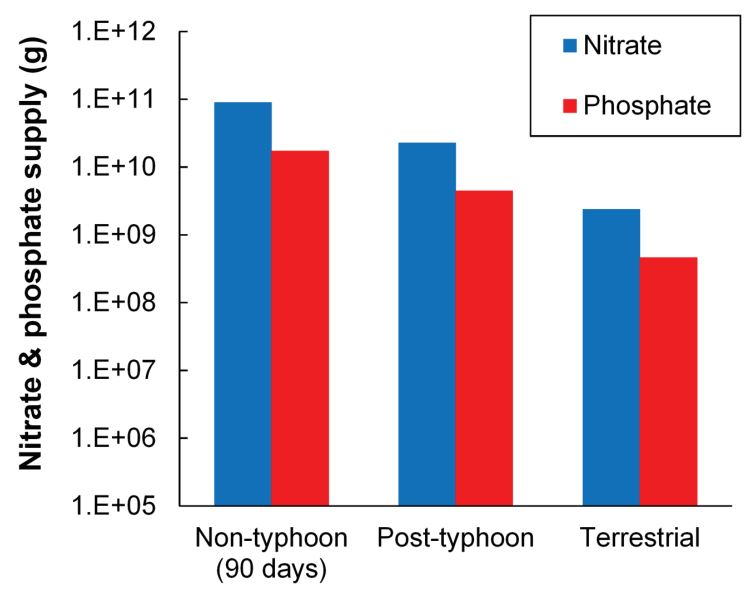

Figure 6. Estimated nitrate and phosphate transport via different sources (e.g. non-typhoon [a period of 90 days], post-typhoon and terrestrial input) to the southern East China Sea in summer.

\section{REFERENCE}

Hung, C.-C., C.-C. Chung, G.-C. Gong, S. Jan, Y. Tsai, K.-S. Chen, W. C. Chou, M.-A. Lee, Y. Chang, M.-H. Chen, W.-R. Yang, C.-J. Tseng and G. Gawarkiewicz, 2013. Nutrient supply in the Southern East China Sea after Typhoon Morakot. J. Mar. Res., 71, 133-149. doi: $10.1357 / 002224013807343425$.

Received: 18 March 2014; revised: 18 March 2014. 